\title{
FamPlex: a resource for entity recognition and relationship resolution of human protein families and complexes in biomedical text mining
}

\author{
John A. Bachman ${ }^{\dagger}$, Benjamin M. Gyori ${ }^{\dagger}$ and Peter K. Sorger ${ }^{*}$ (D)
}

\begin{abstract}
Background: For automated reading of scientific publications to extract useful information about molecular mechanisms it is critical that genes, proteins and other entities be correctly associated with uniform identifiers, a process known as named entity linking or "grounding." Correct grounding is essential for resolving relationships among mined information, curated interaction databases, and biological datasets. The accuracy of this process is largely dependent on the availability of machine-readable resources associating synonyms and abbreviations commonly found in biomedical literature with uniform identifiers.

Results: In a task involving automated reading of 215,000 articles using the REACH event extraction software we found that grounding was disproportionately inaccurate for multi-protein families (e.g., "AKT") and complexes with multiple subunits (e.g."NF- $\kappa \mathrm{B}^{\prime \prime}$ ). To address this problem we constructed FamPlex, a manually curated resource defining protein families and complexes as they are commonly encountered in biomedical text. In FamPlex the gene-level constituents of families and complexes are defined in a flexible format allowing for multi-level, hierarchical membership. To create FamPlex, text strings corresponding to entities were identified empirically from literature and linked manually to uniform identifiers; these identifiers were also mapped to equivalent entries in multiple related databases. FamPlex also includes curated prefix and suffix patterns that improve named entity recognition and event extraction. Evaluation of REACH extractions on a test corpus of $\sim 54,000$ articles showed that FamPlex significantly increased grounding accuracy for families and complexes (from 15 to 71\%). The hierarchical organization of entities in FamPlex also made it possible to integrate otherwise unconnected mechanistic information across families, subfamilies, and individual proteins. Applications of FamPlex to the TRIPS/DRUM reading system and the Biocreative VI Bioentity Normalization Task dataset demonstrated the utility of FamPlex in other settings.

Conclusion: FamPlex is an effective resource for improving named entity recognition, grounding, and relationship resolution in automated reading of biomedical text. The content in FamPlex is available in both tabular and Open Biomedical Ontology formats at https://github.com/sorgerlab/famplex under the Creative Commons CCO license and has been integrated into the TRIPS/DRUM and REACH reading systems.
\end{abstract}

Keywords: Text mining, Protein families, Grounding, Named entity linking, Named entity recognition, Biocuration, Event extraction, Natural language processing 


\section{Background}

A critical challenge in contemporary molecular biology is integrating detailed mechanistic information about specific genes and proteins with genome-scale information about the interaction networks in which these genes participate. Networks of molecular mechanisms are powerful tools for interpreting large-scale data in the context of prior knowledge [1-4]. The construction of biological networks benefits from exchange formats such as BioPAX [5] that allow disparate databases to be aggregated into uniform, machine-readable resources such as Pathway Commons [6]. However, a significant fraction of the information available in the literature has not been recorded in pathway databases. Text mining has the potential to address this gap by augmenting curated network resources with molecular mechanisms automatically extracted from the literature. However, current systems are not yet able to extract mechanisms with a quality equal to that of human curators [7].

One challenge in using text-mined information for biological data analysis is that molecular mechanisms are often described in the literature in terms of aggregate entities such as multi-protein families (e.g., "RAS", "AKT") and multi-subunit complexes (e.g., "NF- $\mathrm{B}$ ", "AP1 ") rather than the specific genes or proteins measured in large-scale experiments. For example, a Pubmed search for "NF-kappaB" yields over 65,000 citations; this transcription factor is not a single molecular entity but rather a class of heterodimers involving combinations of at least five different genes in two families (RELA, RELB, REL, NFKB1, and NFKB2). This poses two challenges for machine reading. First, the text string "NF- $\kappa \mathrm{B}$ " must be normalized to a standard identifier, a task known variously as named entity linking (NEL), named entity normalization (NEN), named entity disambiguation (NED), or simply "grounding." [8]. Second, the mapping of "NF- $\kappa$ " to its constituents must be established so that the activities of NF- $\kappa \mathrm{B}$ can be linked to the properties of the genes from which it is comprised. Such "static relations" must be resolved either by explicit curation or algorithmically [9-11].

Success in the first task, grounding, is essential for practical applications of text mining [12, 13]. Entities without associated identifiers cannot be used for downstream assembly and interpretation tasks, and systematic misidentification of entities clutters extracted networks with errors that skew data analysis. Relevant approaches to grounding have been studied extensively in the context of the general problem of biomedical entity normalization $[8,14-16]$, and generally involve two steps. First, a named entity as encountered in text is normalized, for example by stemming [17], removal of affixes [10], or expansion of abbreviations [16]. Effective preprocessing depends on an explicit or implicit representation of how specific entities (e.g., diseases vs. chemicals vs. genes) variously appear in text (see 2.2.4 in [16]).

The normalized string is then matched to names and synonyms in existing taxonomies [13]. Difficulties in grounding protein families and complexes are encountered in this latter step because there is no standard ontology for these entities as they are commonly described in the scientific literature. Relevant identifiers can be found in protein family databases (InterPro, Pfam, NextProt) and curated interaction databases (Reactome, SIGNOR, OpenBEL) allowing complexes and families to be resolved into their constituent genes. However, such databases generally lack lexical synonyms corresponding to the many ways in which entities are referenced in text, limiting their value for literature mining. Conversely, general biomedical vocabularies and thesauri such as NCIT and MeSH contain entries and lexical synonyms for families and complexes but often lack the ontological resolution of these terms into child concepts (e.g. entries C94701 in NCIT and D055372 in $\mathrm{MeSH}$ for the holo-enzyme AMPK, neither of which define its constituents). In combination, these diverse databases provide substantial information about families and complexes, but integration of this information is difficult because they rarely contain cross-references for related concepts among themselves. Prior work has addressed aspects of normalization for protein families, for example by automatically identifying families and their constituents directly from the literature $[9,15]$ or by combining information in gene family databases with patterns in the names and synonyms of genes [10, 18]. However, the problem of identifying, normalizing, and linking information about protein families and complexes is less well-understood than that of gene normalization $[8,16,18]$, and draws on a smaller base of taxonomic resources.

In this paper we describe FamPlex, a curated lexical and ontological resource that improves grounding and relationship resolution for families and complexes encountered in the mining and curation of biomedical text. FamPlex contains a set of identifiers for protein families and complexes along with mappings that link: (i) text strings and FamPlex identifiers, (ii) FamPlex identifiers and identifiers representing protein families and complexes in other resources, and (iii) FamPlex families/complexes and their constituent members. FamPlex also contains a list of prefixes and suffixes frequently appended to protein names for use in named entity recognition (NER) and entity normalization. The FamPlex resource consists of a set of comma-separated value (CSV) files listing entities and relations, along with Python scripts for checking consistency and identifying equivalent identifiers in other databases. FamPlex is hosted 
on GitHub at https://github.com/sorgerlab/famplex and is made available under the Creative Commons CCO license. It is also available in the Open Biomedical Ontology (OBO) format and can be accessed via the NCBO BioPortal [19] at http://bioportal.bioontology.org/ontologies/ FPLX, or through http://identifiers.org/fplx.

\section{Construction and content}

Development of FamPlex was motivated by an empirical analysis of grounding accuracy in events extracted by the REACH biomedical literature mining software [20, 21]. As described in detail below, we found that grounding of protein families was disproportionately inaccurate and that a relatively small proportion of frequently misgrounded entities accounted for the bulk of all grounding errors. An examination of existing resources highlighted the fragmented nature of information on protein families and complexes and the general lack of suitability of these resources for literature mining. FamPlex was conceived as a "bridging" resource to link available information about families, complexes, and other frequently misgrounded entities across a diverse set of existing bioinformatics databases.

At the core of FamPlex is a set of identifiers representing protein families and complexes (Fig. 1a). FamPlex represents the hierarchical relationships of these high-level entities to each other and to individual genes, along with corresponding synonyms in text and cross-references to other databases where available. Entities and mappings are recorded in a set of CSV files.

\section{Selection of corpus for curation and evaluation}

To empirically guide curation of entities and synonyms based on the frequency of their appearance in literature we selected a corpus of articles focused on the proteins, protein families, complexes, and molecular events relevant to pathway biocuration (Fig. 1b). Specifically, we combined the 3752 signaling proteins in Reactome [22] with the members of protein families and complexes defined in OpenBEL resource files [23]. From this gene list a corpus of 269,489 papers was assembled by retrieving papers curated for each gene from the Entrez Gene database [24]. Abstracts were obtained from MEDLINE and full texts were downloaded either from the Pubmed Central Open Access subset (in XML or text format), the Pubmed Central Author Manuscript Collection, or via the Elsevier text and data mining API (Table 1).

\section{Event extraction from text using REACH and INDRA}

The corpus of $\sim 270,000$ papers was processed with the $\mathrm{REACH}$ event extraction software [21], yielding a set of sentences, named entities, and extracted relations (Fig. 1b). REACH is built on widely-used methods for syntactic parsing and named-entity recognition: it uses the Stanford CoreNLP parser [25] for syntactic parsing and draws information on biology-specific named entities from Uniprot, InterPro, Pfam, HMDB, ChEBI, Gene Ontology, MeSH, Cellosaurus, ATCC, and CellOntology. As a final step we used the INDRA software [26] to convert events extracted by REACH into INDRA Statements, a format suitable for analyzing and assembling sets

a

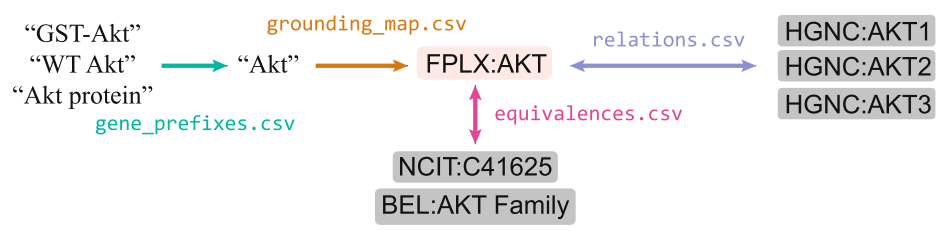

b

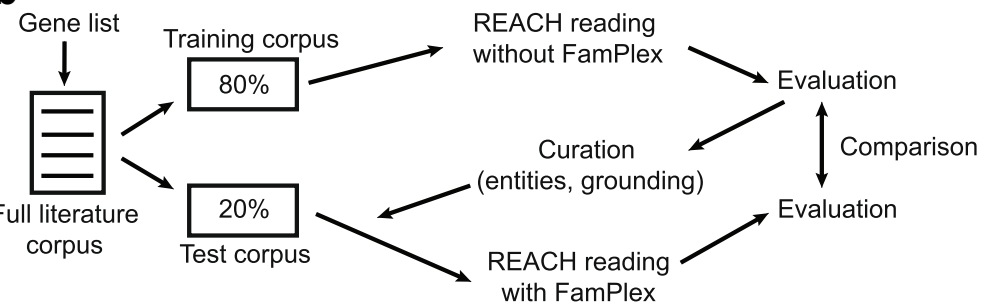

Fig. 1 FamPlex links named entities to protein families and complexes and their constituents. a Structure of FamPlex content. The affixes in gene_prefixes. csv can be used to improve recognition of molecular entity names, which can be linked to database identifiers using the lexical synonyms in grounding_map. csv. FamPlex itself contains identifiers representing families and complexes which are mapped to corresponding identifiers in other databases in equivalences. CSv. Hierarchical relationships among families, complexes, and genes are listed in relations. csv. b Workflow for curation and evaluation. A gene list was used to define a corpus of articles that was divided into two subsets, "training" and "test". The "training" corpus was processed with REACH and results were evaluated and used to guide curation. The "test" corpus was processed after incorporation of FamPlex and results were compared against the baseline from the training corpus 
Table 1 Composition of article corpus by source

\begin{tabular}{lrr}
\hline Text source type & Number & $\%$ \\
\hline MEDLINE Abstract & 183,019 & 67.9 \\
Elsevier XML & 40,261 & 14.9 \\
Pubmed Central Open Access Subset XML & 32,113 & 11.9 \\
Pubmed Central Author Manuscript XML & 13,777 & 5.1 \\
No content retrieved & 267 & 0.1 \\
Pubmed Central Open Access Subset text file & 52 & 0.02 \\
Total & 269,489 & 100.0 \\
\hline
\end{tabular}

of mechanisms into networks and executable models of various kinds.

\section{Characterizing patterns of grounding errors}

The set of entities and events extracted by REACH was used to characterize patterns of grounding errors and prioritize entities and their lexical synonyms for subsequent curation (Fig. 1b). Prior to curation, the corpus of articles was divided into two sets: a "training" set and a "test" set consisting of $80 \%(215,360)$ and $20 \%(53,840)$ of the articles, respectively (the total of 269,200 articles for the combined training and test sets differs from the full corpus of 269,489 reported in Table 1 due to the fact that content could not be retrieved for 267 articles and reading failed for an additional 22). The "training" set of articles was processed with REACH in the absence of FamPlex to evaluate baseline grounding accuracy and guide curation. Following curation, the "test" set of articles was processed with a version of REACH incorporating FamPlex. The partitioning of articles was performed to ensure that estimates of grounding accuracy would not be biased toward the specific set of articles used for curation.

\section{Definition of protein families and complexes and their constituents}

Identifiers for protein families and complexes in FamPlex were created by drawing on two resources: 1) identifiers created de novo in FamPlex to correspond to named entities encountered in event extraction, and 2) identifiers drawn from the OpenBEL resource. In the first case, identifiers were prioritized by their frequency of occurrence among extracted events, with common entities such as "NF-kappaB", "Ras", "PI3kinase", "Akt", etc., accounting for a significant fraction of grounding errors. In the case of OpenBEL, identifiers for protein families and complexes were drawn from the resource files protein-families.xbel and named-complexes.xbel, accessible via the OpenBEL GitHub repository at https://github.com/OpenBEL/ openbel-framework-resources. The full list of all FamPlex identifiers is contained in the text file entities. csv.
Members of protein families and complexes are enumerated in the file relations.csv using two types of relations: is a and partof, denoting membership in a family or a complex, respectively (Fig. 1a). These relationships can be applied hierarchically to describe multi-level protein subfamily relationships or protein complexes that are hetero-oligomers of subunits belonging to distinct families (Fig. 2a). For example, 5' AMP-activated protein kinase, or AMPK, is a heterotrimeric protein consisting of alpha, beta, and gamma subunits: the alpha and beta subunits comprise families with two isoforms each, and the gamma subunit family has three isoforms. This hierarchical structure can be represented in FamPlex by using a combination of isa and partof relationships to link the identifiers for the subunit genes to FamPlex-specific identifiers for the subunit families and the full complex (Fig. 2a).

Information on protein family and complex membership was drawn from OpenBEL resource files, HGNC, Reactome, InterPro, and Wikipedia, and manually curated for consistency. Where there were discrepancies among sources about family or complex membership we prioritized what we judged to be the most common usage. For example, the InterPro entry corresponding to the Ras protein family (IPR020849) lists 145 human proteins as members, whereas usage in literature and interaction databases recognizes only KRAS, NRAS, and HRAS as family members.

\section{Mapping FamPlex identifiers to related resources}

Entities defined in FamPlex are cross-referenced to corresponding identifiers in other databases and ontologies in the equivalences file (equivalences.csv; Fig. 1a). Figure $2 b$ shows the subsets of FamPlex identifiers containing mappings to different types of external databases: databases of interactions curated from literature (OpenBEL, Reactome), databases containing specific information about protein families and complexes (Pfam, InterPro, NextProt, and Gene Ontology), and generalpurpose biomedical vocabularies (NCIT, MeSH). There are 32 unmapped entries for which no equivalent entry was found in external databases; these entries are implicitly defined in FamPlex by the specific genes that they contain as members.

Identifier mappings between FamPlex and Reactome and InterPro were obtained in a semi-automated fashion. The gene-level members of each FamPlex family and complex were used to query Reactome and InterPro for families and complexes containing these genes. Reactome and InterPro families with equivalent sets of members were incorporated into equivalences. csv. Python scripts for generating and updating these mappings are available in the FamPlex GitHub repository at import/reactome_mappings.py and 

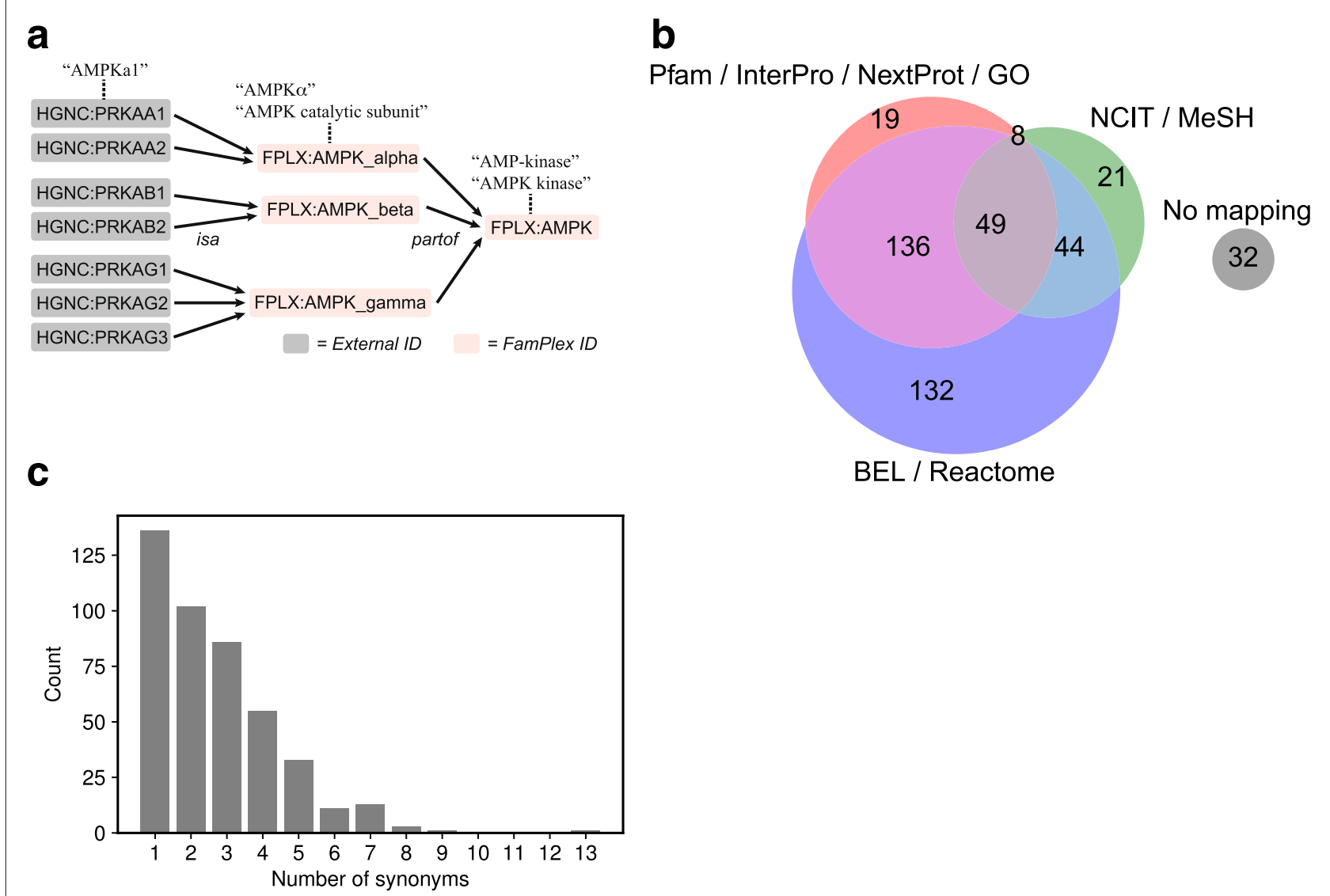

Fig. 2 FamPlex links identifiers for families and complexes to members, other databases, and lexical synonyms. a FamPlex uses isa and partof predicates to represent the hierarchical relationships between specific genes, families and complexes. Lexical synonyms can be associated with entities at each level. b Mappings of FamPlex identifiers to outside databases. c Number of lexical synonyms curated for FamPlex identifiers in the grounding map

import/interpro_mappings.py. Additional identifier mappings to Pfam, NCIT, NextProt, GO and MeSH were collected by entering FamPlex identifiers and lexicalizations into the TRIPS/DRUM web service available at http://trips.ihmc.us/parser/cgi/drum [27]. The TRIPS/DRUM web service returned identifier mappings and their scores based on partial string matches to a variety of databases, which were then manually curated for inclusion in FamPlex.

\section{Curation of lexical synonyms for entities}

Entities defined in FamPlex are associated with lexical synonyms in the grounding map (grounding map.csv; Fig. 1a). These synonyms allow natural language processing tools to match named entities extracted from text to the protein families and complexes contained in the FamPlex hierarchy.

Lexical synonyms were curated in two ways. First, named entities extracted from the "training" articles read by REACH were sorted by frequency, and named entities corresponding to FamPlex families and complexes were added to the grounding map. Entries were also added to the grounding map for frequently occurring but incorrectly grounded named entities of other types (e.g., proteins, chemicals, and biological processes). For less-frequently encountered families and complexes, synonyms were curated using a different approach: names and synonyms for the gene-level members of families and complexes were used to search the named entities extracted by REACH. Potential matches were identified by fuzzy string matching (Levenshtein distance [28]) using the Python fuzzywuzzy package and subsequently manually curated.

Of the 2413 entries in the FamPlex grounding map, 1458 map to FamPlex identifiers; the remaining 955 map to frequently occurring proteins, chemicals, and biological processes. The distribution of lexical synonyms across the set of FamPlex identifiers is shown in Fig. 2c. The frequently-occurring entities NFkappaB and ERK have the most synonyms, with 13 and 9, respectively; many other less-frequently occurring entities have only a single synonym. Examples of synonyms for NFkappaB include 
"NF-kB", "NFkappaB", and "NF-kappaB TFs"; synonyms for ERK include "ERK 1/2", "ERKs", and "Extracellular Signal Regulated Kinase".

\section{Curation of gene/protein affixes}

References to genes and proteins in the literature are often modified by affixes that describe modifications or other context. For example, "mmu-AKT1" and "pAKT1" refer to murine and phosphorylated AKT1, respectively. A list of 137 case-sensitive affixes was tabulated by alphabetically sorting a list of $\sim 80,000$ named entities resulting from event extraction and manually identifying common affix patterns. These affixes were subsequently grouped into six semantic categories (Table 2). The largest category, "experimental context", contains affixes used to identify the precise variant of a gene used in an experiment; these often refer to protein tags or gene delivery methods. Two of the six categories affect event extraction as well as grounding: "protein state" affixes contain information on modification, location and mutation states, while "inhibition" affixes invert the apparent polarity of an extracted event. For example, a positive regulation event mediated by "BRAF siRNA" actually represents a negative regulation by BRAF itself. The full list of affixes can be found in the CSV file gene_prefixes.csv (Fig. 1a).

\section{Resource structure and scope}

FamPlex comprises 441 families and complexes that together cover 2040 specific genes through isa and partof relations. Most FamPlex entries (315) are at the top level of the hierarchy, having no parent entities; 111 entries are at an intermediate level, having both parent and child entities; 15 entities function as placeholders with no parent or child relations currently specified. This latter category consists primarily of functional categories with many potential protein members, e.g., GTPase, Phosphatase, Protease, etc.

The top-level entries vary in terms of the depth of the hierarchy they subsume with the majority of entries (275 in total, two examples being RAS and RAF) directly being resolved to a set of specific constituent genes. 37 entries have two subsumed levels (for instance PLC

Table 2 Gene/protein affix types

\begin{tabular}{lrl}
\hline Category & \# of affixes & Example \\
\hline Experimental context & 63 & eGFP- $\{$ Gene name $\}$ \\
Protein state & 30 & phospho- $\{$ Gene name $\}$ \\
Inhibition & 22 & shRNA- $\{$ Gene name $\}$ \\
Generic descriptor & 12 & $\begin{array}{l}\text { proto-oncogene } \\
\text { Gene name }\}\end{array}$ \\
Species & 9 & mmu- $\{$ Gene name $\}$ \\
mRNA grounding & 1 & $\{$ Gene name $\}$ mRNA \\
\hline
\end{tabular}

which subsumes the subfamilies PLCD, PLCG, and PLCB, which in turn subsume a total of nine constituent genes), and 3 entries (G_protein, HSP9O and PI3K) subsume three levels.

FamPlex entries vary in terms of the number of children they subsume with an average of $6.0 \pm 7.1$ children, the large standard deviation indicating the long-tailed nature of the distribution. While the median FamPlex entry has 3 children, several entries have a much larger number, including RAB (68 children), Histone (60 children) and Cyclin (31 children).

To characterize the scope and relevance of the different identifiers we quantified the prevalence of each FamPlex entry in PubMed-indexed articles. We conducted PubMed searches for each lexicalization of a given FamPlex entry (using the relatively restrictive "text word" search mode of PubMed to avoid partial matches and matches to meta-information) and counted the total number of unique articles found for each FamPlex entry itself and also for each entry and all its children. The total number of PubMed-indexed articles mentioning one or more FamPlex entries (or children) was $4,012,468$, or roughly $14 \%$ of all PubMed-indexed literature. The mean number of citations per FamPlex entry was 13,091 $\pm 26,733$ with a median of 3034, reflecting a distribution skewed toward a small number of highly cited entries. When including the children of each entry, the number of citations per entry was higher, with a mean of $16,136 \pm 29,491$ and a median of 4929 . The most commonly appearing FamPlex entry was Interferon with 204,228 associated articles; only 11 FamPlex entries had fewer than 100 associated PubMed citations. Thus, FamPlex covers entities that are frequently mentioned in the biomedical literature.

\section{Utility and discussion}

\section{Protein families and complexes appear frequently in} events extracted from literature and are often incorrectly grounded

To evaluate baseline grounding performance without FamPlex we manually scored a random sample of 300 named entities generated by running $\mathrm{REACH}$ on the training corpus. Entities were categorized by type (protein/gene, family/complex, small molecule, biological process, microRNA, and other/unknown) and the database mappings identified by REACH were scored for correctness (Table 3). Where the entity text alone was insufficient to evaluate grounding accuracy, the sentence in which the entity was embedded was examined in the context of the original paper.

We found that references to protein families and complexes were second only to genes and proteins in the frequency of their occurrence in events extracted from text, accounting for $17.7 \%$ of all extracted entities 
Table 3 Entity frequency and grounding accuracy for 300 entities, with and without FamPlex

\begin{tabular}{|c|c|c|c|c|c|c|c|c|}
\hline & \multicolumn{4}{|c|}{ No FamPlex } & \multicolumn{4}{|c|}{ With FamPlex } \\
\hline & $\#$ & Entity \% & \# Corr. & $\%$ Corr. & $\#$ & Entity \% & \# Corr. & $\%$ Corr. \\
\hline Protein/gene & 169 & 56.3 & 133 & $78.7 \pm 3.1$ & 172 & 57.3 & 154 & $89.5 \pm 2.3$ \\
\hline Family/complex & 53 & 17.7 & 8 & $15.1 \pm 4.9$ & 52 & 17.3 & 37 & $71.2 \pm 6.3$ \\
\hline Small molecule & 33 & 11.0 & 18 & $54.5 \pm 8.7$ & 26 & 8.7 & 14 & $53.8 \pm 9.8$ \\
\hline Biological process & 28 & 9.3 & 24 & $85.7 \pm 6.6$ & 28 & 9.3 & 28 & $100.0 \pm 0.0$ \\
\hline Other/unknown & 16 & 5.3 & 0 & $0.0 \pm 0.0$ & 21 & 7.0 & 0 & $0.0 \pm 0.0$ \\
\hline microRNA & 1 & 0.3 & 0 & $0.0 \pm 0.0$ & 1 & 0.3 & 0 & $0.0 \pm 0.0$ \\
\hline
\end{tabular}

Standard error was calculated using the formula $\sqrt{(k / n)(1-k / n) / n}$ where $k$ is the number of samples in the given category and $n$ is the total number of samples Row 1: significant with $p<0.01$

Row 2: significant with $p<10^{-8}$

(Table 3). Grounding accuracy was substantially lower for families and complexes relative to genes and proteins, with only $15.1 \%$ of families and complexes correctly grounded compared to $78.7 \%$ for individual proteins (Table 3 ). The $15 \%$ rate of correct grounding for families and complexes reflected accurate matches to identifiers in InterPro or Pfam. Notably, seven of the top ten most frequently occurring ungrounded entity texts in the training corpus represented families or complexes ("NF-kappaB", "ERK1/2", "mTORC1", "NFkappaB”, "PDGF", "IKK", and "histone H3"; Table 4). Overall, REACH identified a total of 163,428 unique named entity strings involved in events, out of which 2873 were grounded (correctly or incorrectly) to a protein family or complex (1.8\%).

Close inspection of errors made by $\mathrm{REACH}$ in grounding frequently-occurring families and complexes in the absence of FamPlex revealed the causes of both missing and incorrect groundings. Missing groundings occurred when named entities for families and complexes had no corresponding identifiers or synonyms in any of the indexed databases. This was true of the entity "Ras", as well as the most frequently occurring family-level entity, "NF-kappaB".

On the other hand, incorrect grounding of family-level entities occurred due to exact (but spurious) matches to obscure synonyms for other genes listed in Uniprot or HGNC. In some cases these genes were unrelated to the family but had synonyms shadowing the family name: for example, "ERK" and "Cyclin" were grounded to the human genes EPHB2 (Uniprot P29323) and PCNA (Uniprot P12004) due to the presence of these strings as synonyms. Another class of grounding errors involved the matching of a string representing the basename of a human protein family to the single ortholog of the family in a different organism. Representative examples include the misgrounding of "AKT" to the Dictyostelium discoideum gene $p k b A$ and of "JNK" to the Drosophila melanogaster gene $b s k$, both of these listing the human gene family name as synonyms.

The most common ungrounded strings (those in the highest percentile by frequency of occurrence) accounted for a surprisingly large proportion of the overall number of ungrounded string occurrences, as shown by the orange

Table 4 Top 10 most frequently occurring ungrounded entity texts with and without FamPlex in the training and test corpora, respectively

\begin{tabular}{|c|c|c|c|c|c|c|}
\hline \multirow[b]{2}{*}{ Rank } & \multicolumn{3}{|l|}{ No FamPlex } & \multicolumn{3}{|l|}{ With FamPlex } \\
\hline & Entity Text & Count & $\%$ of Total & Entity Text & Count & $\%$ of Total \\
\hline 1 & NF-kappaB & 18,381 & 3.78 & PKCzeta & 222 & 0.24 \\
\hline 2 & ERK $1 / 2$ & 6137 & 1.26 & RANTES & 176 & 0.19 \\
\hline 3 & mTORC1 & 2753 & 0.57 & $D C$ & 169 & 0.18 \\
\hline 4 & NFkappaB & 2425 & 0.50 & LDL & 168 & 0.18 \\
\hline 5 & c-Jun & 2369 & 0.49 & $\lg \mathrm{E}$ & 152 & 0.17 \\
\hline 6 & antigen & 1724 & 0.35 & SDF-1-alpha & 141 & 0.15 \\
\hline 7 & PDGF & 1626 & 0.33 & receptor & 128 & 0.14 \\
\hline 8 & IKK & 1542 & 0.32 & beta1 integrin & 127 & 0.14 \\
\hline 9 & $\mathrm{c}-\mathrm{SrC}$ & 1362 & 0.28 & p38alpha & 126 & 0.14 \\
\hline 10 & histone H3 & 1347 & 0.28 & CD4+ & 124 & 0.14 \\
\hline
\end{tabular}


curve in Fig. 3a. The deviation of this curve from a uniform distribution (shown by the dotted gray line in Fig. 3a) arises because the empirical distribution of ungrounded entities is highly skewed, with a small number of very common entities accounting for a large percentage of occurrences. For example, half of all ungrounded string occurrences in the training corpus involved the top $2.4 \%$ most frequently occurring strings (2666 distinct strings). This explains why curation that is focused specifically on frequently occurring misgrounded entities has the potential to substantially improve overall grounding and reading performance.

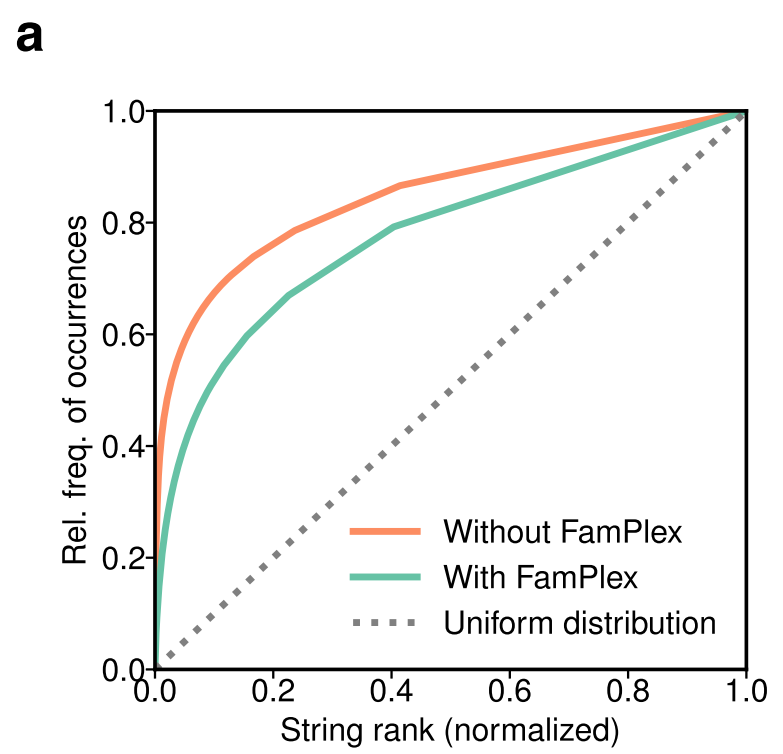

b

C
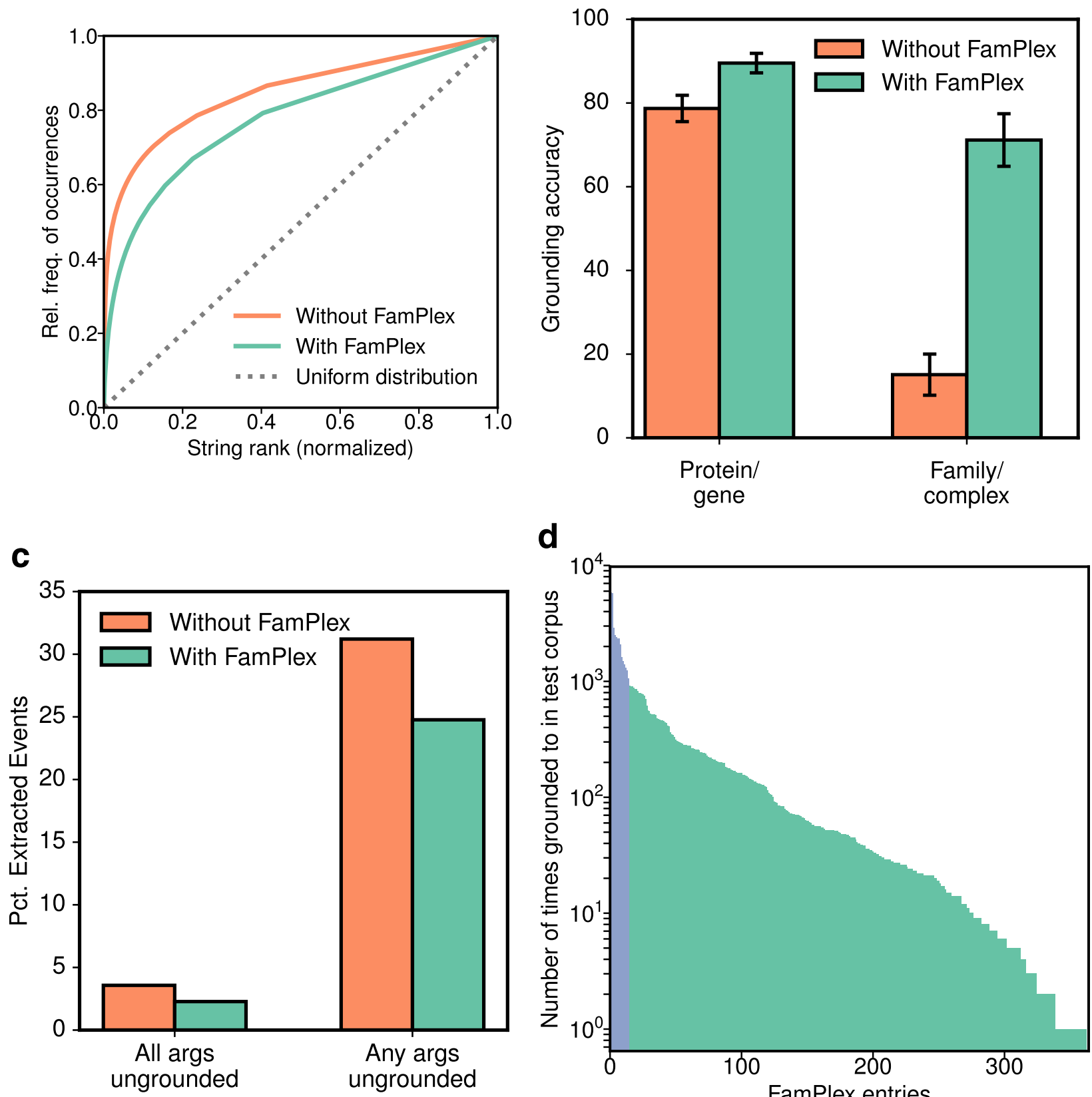

d

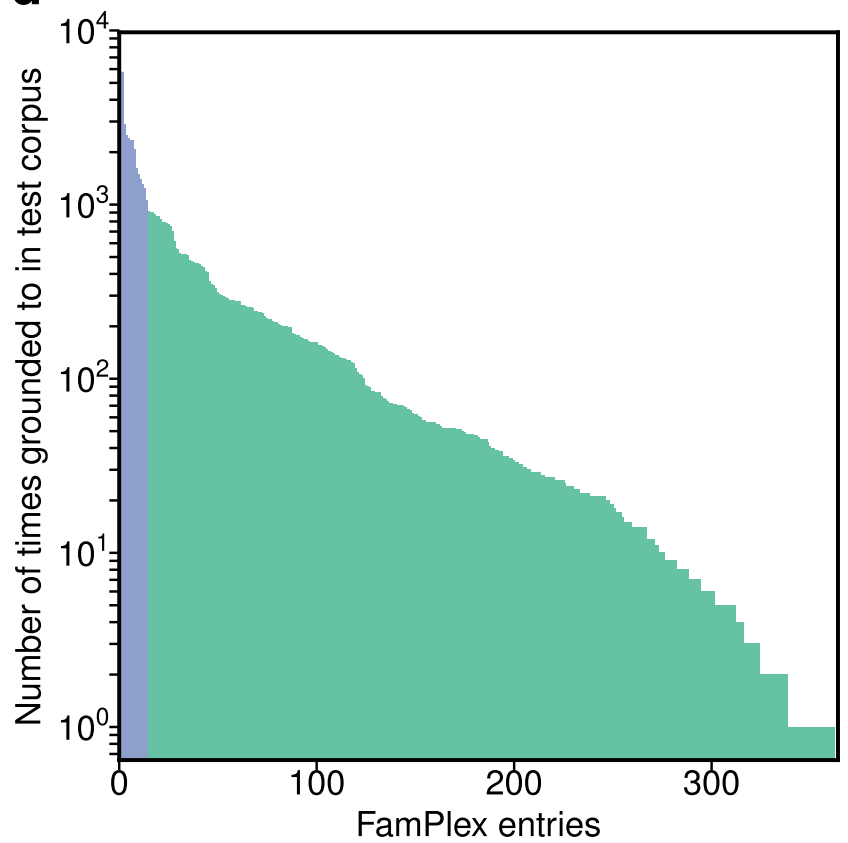

Fig. 3 FamPlex improves grounding accuracy. a Cumulative occurrences of ungrounded entities by frequency of the entity text. Deviation from the dotted gray line, representing a uniform frequency distribution, indicates the extent to which a small number of frequently occurring entities account for a disproportionate share of missed groundings. b Improvements in grounding accuracy for proteins/genes and families/complexes, with and without the use of FamPlex. c Reduction in the proportion of extracted events containing ungrounded entities, with and without FamPlex. $\mathbf{d}$ Number of groundings to FamPlex identifiers in the test corpus. The 15 most frequent identifiers account for $50 \%$ of all groundings and are shown in blue 


\section{Use of FamPlex in text mining improves grounding and relationship resolution for protein families and complexes in two event extraction systems}

Following the manual curation of FamPlex identifiers and associated synonyms and the integration of FamPlex into REACH and INDRA, we performed a second evaluation on a random sample of 300 named entities drawn from the results of processing the test corpus (Table 3). The frequency of entity types was comparable between the training and test samples, with proteins/genes and families/complexes accounting for roughly three-quarters of all entities. Improvements in grounding were substantial for both classes, with grounding accuracy for families and complexes rising from 15 to $71 \%$ (Fig. 3b; Table 3). Grounding accuracy for proteins and genes increased from 79 to $90 \%$, an improvement attributable to the curation of synonyms for frequently occurring proteins. With the incorporation of FamPlex, the overall percentage of unique entity strings grounded to protein family or complex identifiers doubled relative to the training corpus, with REACH grounding 2080 of 57,088 unique entities to a FamPlex, InterPro or Pfam identifier (3.6\%).

An analysis of the distribution of the remaining ungrounded entities showed that FamPlex addressed a substantial proportion of the most frequently occurring grounding failures (Fig. 3a, green curve). As shown in Table 4, the top ten most frequently occurring ungrounded entities in the test set occur at a lower overall frequency and include a functional category ("receptor") but no specific protein families or complexes. To examine the impact of grounding improvements at the level of extracted events, we calculated the proportion of events consisting either of any or all ungrounded entities, and found that both metrics improved with the use of FamPlex (Fig. 3c). These measures, which deal only with event entities that were ungrounded, represent an underestimate of the overall improvement in grounding because they do not account for cases in which entities were grounded to the wrong identifier in the absence of FamPlex.

To characterize whether improvements in grounding were driven by a small subset of frequently-occurring entities in FamPlex or were more broadly distributed across families and complexes, we counted the occurrences of mappings to each FamPlex identifier in events extracted from the test corpus. We found that the 15 most frequently-referenced FamPlex identifiers accounted for $50 \%$ of all FamPlex groundings (blue bars in Fig. 3d); the top five are shown in Table 5. At the same time, 363 of the 441 FamPlex identifiers were mapped to text at least once, suggesting that the great majority of identifiers and lexical synonyms in FamPlex are useful for improving grounding (Fig. 3d).

As a second means to evaluate FamPlex we used the TRIPS/DRUM reading system [27]. Unlike REACH,
Table 5 FamPlex entries most frequently grounded to in test corpus, with the absolute number of times grounded to in the test corpus and the percentage normalized to all FamPlex groundings

\begin{tabular}{lrr}
\hline & $\#$ & \% FamPlex \\
\hline ERK & 6301 & 7.6 \\
AKT & 5839 & 7.1 \\
NFkappaB & 5768 & 7.0 \\
TGFB & 2877 & 3.5 \\
Pl3K & 2486 & 3.0 \\
\hline
\end{tabular}

which uses strict string matching against a set of dictionaries, TRIPS uses soft matching to provide a ranked, scored list of groundings for each named entity. Relevant dictionaries used by TRIPS include Pfam and NextProt for protein families, GO for protein complexes and NCIT for both.

We compiled two versions of TRIPS, one in which FamPlex was included as a grounding resource, and one in which it was omitted. Since the throughput of TRIPS is substantially lower than that of REACH, we selected a random sample of 100 abstracts from the combined training and test set for reading with and without FamPlex. We then manually curated 500 randomly sampled entities appearing in TRIPS extractions, determining whether each entity represented a protein family or complex, and if so, whether: (i) the top scoring grounding match was correct, and (ii) any of the grounding matches were correct. In contrast to our evaluation of entity grounding in $\mathrm{REACH}$, in which the curated entities were limited to arguments of events, here we considered all entities identified in text by TRIPS as candidate families or complexes for curation. This broader pool of candidate entities included names of cell lines, organisms, biological processes, etc., and therefore also a smaller proportion of molecular entities such as families and complexes.

In the case of TRIPS without FamPlex, 36 of 500 entities sampled from the TRIPS output corresponded to families or complexes. Of these, we found that the top scoring grounding was correct for 23 (64\%); 29 entities (81\%) had at least one correct grounding. The higher baseline accuracy of family/complex grounding in comparison with REACH likely reflects broader coverage of relevant identifiers due to the inclusion of NextProt and NCIT (used by TRIPS but not by REACH) and the more robust but computationally costly soft-matching and ranking procedure used for grounding. While no single resource accounted for the majority of all matches, top-scoring matches were roughly equally distributed between NCIT and NextProt. Moreover, of the 17 entities that were correctly grounded in NCIT, 7 (41\%) had no identified child concepts, making it impossible to link these families and complexes to 
constituent genes. Thus, while TRIPS was more successful than REACH at finding relevant groundings for families and complexes in the absence of FamPlex, the multiplicity of alternative groundings and the unresolved nature of these terms in the ontologies used posed a distinct problem, that of relationship resolution.

Incorporating FamPlex into TRIPS improved both the accuracy and consistency of grounding. In a sample of 500 entities extracted by TRIPS using FamPlex, 33 corresponded to families and complexes; the top-scoring grounding was correct for $26(79 \%)$ of these and a further four (91\% overall) had at least one correct grounding. While the small sample sizes limit quantitative conclusions about the degree of improvement, we noted that in 18 of $26(69 \%)$ cases in which the top-scoring grounding was correct, it was grounded to a FamPlex identifier, and in 20 of 26 (77\%) a FamPlex grounding was among the top two matches. This indicates that FamPlex identifiers and lexicalizations have a higher coverage for families and complexes encountered in text by TRIPS than other resources used, allowing for more consistent relationship resolution and integration of information.

\section{FamPlex includes a large majority of families and complexes annotated by human curators in text}

In addition to the evaluations of grounding precision described above, we sought to establish a measure of the recall of FamPlex in terms of its coverage of relevant families and complexes in a manually curated dataset. Evaluations solely against machine reading output, as described above, do not provide a true recall measure because the readers extract only a subset of the events and entities from the underlying text.

To evaluate recall we used the dataset prepared for the bioentity normalization task from Biocreative VI Task 1.1 (http://www.biocreative.org/tasks/biocreativevi/track-1/). The dataset, drawn from the EMBO SourceData annotation project [29], contains a corpus of entity text strings from figure legends in published papers, most of which have been annotated with database identifiers by human curators. Our aim was to evaluate the extent to which FamPlex incorporates identifiers and lexicalizations for the family and complex-level entities identified in text by human curators.

Inspection of the Biocreative dataset revealed that curators annotated family- and complex-level strings in multiple ways: to a single gene, multiple genes, or simply left ungrounded. We therefore partitioned the annotation data into multiple subsets for the purposes of evaluation (Table 6). The first of these was the subset of 19,228 entities grounded to human Uniprot or NCBI gene identifiers, which we denote Annotation Subset 1 (AS1; $18.7 \%$ of the total). Of these, 2439 entities (2.4\% overall) were grounded to multiple human gene or protein identifiers; these therefore correspond to gene families or protein complexes (denoted AS2). We also drew from "ungrounded" entities, i.e., annotations labeled "gene" or "protein" but lacking identifiers. A large majority of these represented experimental elements or protein tags, e.g. "GFP", "FLAG", "GST", etc. To streamline curation, we filtered ungrounded entities against the affixes included in FamPlex; a high proportion of ungrounded entities (8250 of 14,227 , or $58 \%$ ) had matches in the FamPlex affixes list in gene_prefixes.CSV, leaving 5977 entities for further curation, a subset denoted AS3 (Table 6).

An initial round of scoring focused exclusively on identifying the proportion of the 2439 entities in AS2 (the subset containing multiple gene/protein groundings) covered by FamPlex; we found that 1908 (78\%) had case-insensitive matches in the FamPlex grounding map. Of the remaining 531 unmatched entities (representing 109 unique strings), manual curation indicated that 51 corresponded to noncoding RNAs and were excluded, leaving 2388 entities $(1908+480)$ with multiple gene/protein groundings. Of the remaining 480 entities representing proteins, manual curation indicated that 97 had corresponding identifiers in FamPlex. We therefore calculated that FamPlex contained both string matches and identifiers for $79.9 \%$ of the entity texts in AS2, and identifiers but not string matches for a slightly higher proportion (84\%; Table 7).

Because families were not always grounded to multiple gene/protein identifiers by human curators, we performed

Table 6 Subsets of the Biocreative VI entity normalization dataset relevant to the FamPlex evaluation

\begin{tabular}{lrr}
\hline Annotation category & $\#$ & \% of total \\
\hline All annotations & 102,717 & 100.0 \\
Grounded to gene/protein & 44,576 & 43.4 \\
Grounded to human gene/protein (AS1) & 19,228 & 18.7 \\
Grounded to multiple human genes/proteins (AS2) & 2439 & 2.4 \\
Ungrounded gene/protein & 14,227 & 13.9 \\
Ungrounded gene/protein matching FamPlex affix & 8250 & 8.0 \\
Ungrounded gene/protein not matching FamPlex affix (AS3) & 5977 & 5.8 \\
\hline
\end{tabular}

Entities evaluated against FamPlex were drawn from the categories highlighted in bold 
Table 7 Extent of FamPlex family/complex coverage evaluated against subsets of the Biocreative VI entity normalization dataset

\begin{tabular}{lrr}
\hline Annotations scored & String matches & Corresponding IDs \\
\hline Multiple gene/protein groundings (AS2) & $1908 / 2388$ (79.9\%) & $2005 / 2388(84.0 \%)$ \\
Families curated from random sample of AS1 + AS3 & $89 / 109(81.7 \%)$ & $96 / 109(88.1 \%)$
\end{tabular}

a second evaluation in which we manually curated a random sample of entities drawn from AS1 + AS3. Of 764 curated entity strings, 109 were found to be synonyms for protein families or complexes (note that, unlike in the evaluation against AS2 above, this assessment was made independently of the annotations contained in the dataset). As in the previous evaluation, these were scored for the presence of string matches and/or corresponding IDs in FamPlex, yielding similar figures of 81.7 and $88.1 \%$, respectively (Table 7 ). Taken together, these results demonstrate that FamPlex incorporates identifiers and lexical synonyms for a large proportion of the families and complexes relevant to manual biocuration tasks from literature.

\section{FamPlex resolves hierarchical relationships in extracted events}

A key feature of FamPlex is that it allows for relationship resolution not only "horizontally" (between different databases) but also "vertically" (between genes, families, complexes, and any intermediate sets involving these elements). Lexical synonyms can be defined at all levels in the FamPlex hierarchy (Fig. 2a). The combination of a hierarchical representation with a mapping of entities to text at each level allows information about biological interactions to be correctly organized and cross-referenced.

For example, the FamPlex family PLC, representing the family of phospholipase $\mathrm{C}$ enzymes, contains both individual genes (e.g., PLCE1) and FamPlex subfamilies (e.g., PLCG, a sub-family consisting of the genes PLCG1 and PLCG2) as members (Fig. 4a). In results from the test corpus we found descriptions of meaningful biochemical mechanisms associated with all three levels of this hierarchy-family, subfamily, and genes (Fig. 4a). Moreover, relevant events were extracted for 12 of the 15 entities in the phospholipase $C$ entity hierarchy, demonstrating the diversity of available mechanistic information and the importance of relationship resolution.

To characterize the relevance of multi-level relationship resolution more broadly, we counted the number of times a named entity identified by $\mathrm{REACH}$ in the test corpus was mapped to a FamPlex identifier at three or more hierarchical levels: the gene level (lowest), the top-level family or complex (highest), and any intermediate level. Distributions of groundings for five FamPlex entries with three or more entity levels are shown in Fig. 4b. Overall, we found that 33 top-level FamPlex entries (i.e. ones that are not subsumed through an isa or partof relation by another FamPlex entry) were associated with groundings at three or more distinct levels, and 242 top-level FamPlex entries had groundings at two levels (i.e. grounding to the FamPlex entry itself and its constituent genes), showing that gene functions are commonly discussed across multiple levels of specificity.

We also found that the identifier level used most frequently for grounding differed among protein families and complexes, limiting generalizations about the relative priority of gene- vs. family-level grounding for event extraction. For example, for AMPK, the majority of references in the literature were to the top-level AMPK complex, with a relatively small fraction of references to constituent genes or intermediates. On the other hand, most mappings to the family representing Phospholipase C (PLC in FamPlex) were to constituent genes such as PLCG1, PLCD1, etc. Finally, for the family of Activins (hetero- and homodimers of the transforming growth factor beta family, Activin in FamPlex), most references were to specific dimer subtypes-Activin A, Activin AB and Activin Bwhich are found at an intermediate level in the FamPlex hierarchy.

\section{Comparison of FamPlex with other resources}

FamPlex bears similarities to three types of existing resources. The first of these are large, systematic assemblies of protein families derived from sequence and domain analysis; this set includes Pfam, InterPro, and Homologene. As a curated resource, FamPlex is less comprehensive, since it includes only human genes and focuses primarily on gene families and lexicalizations that are described in existing literature. However, FamPlex includes complexes as well as families, based on the observation that these high-level groupings of proteins are often interwoven in discussions of gene function (e.g., "AMPK" and "AMPK-alpha"; Fig. 2a). FamPlex also provides lexical synonyms for families and complexes, a feature generally absent from large protein family databases.

A second class of comparable resources are the taxonomies of protein families and complexes defined as part of biocuration projects or tools; examples include Reactome, SIGNOR, and OpenBEL. These taxonomies are designed to meet the need of biocurators to specify mechanistic interactions at the family or complex level. Of these resources, we found the families and complexes defined by 


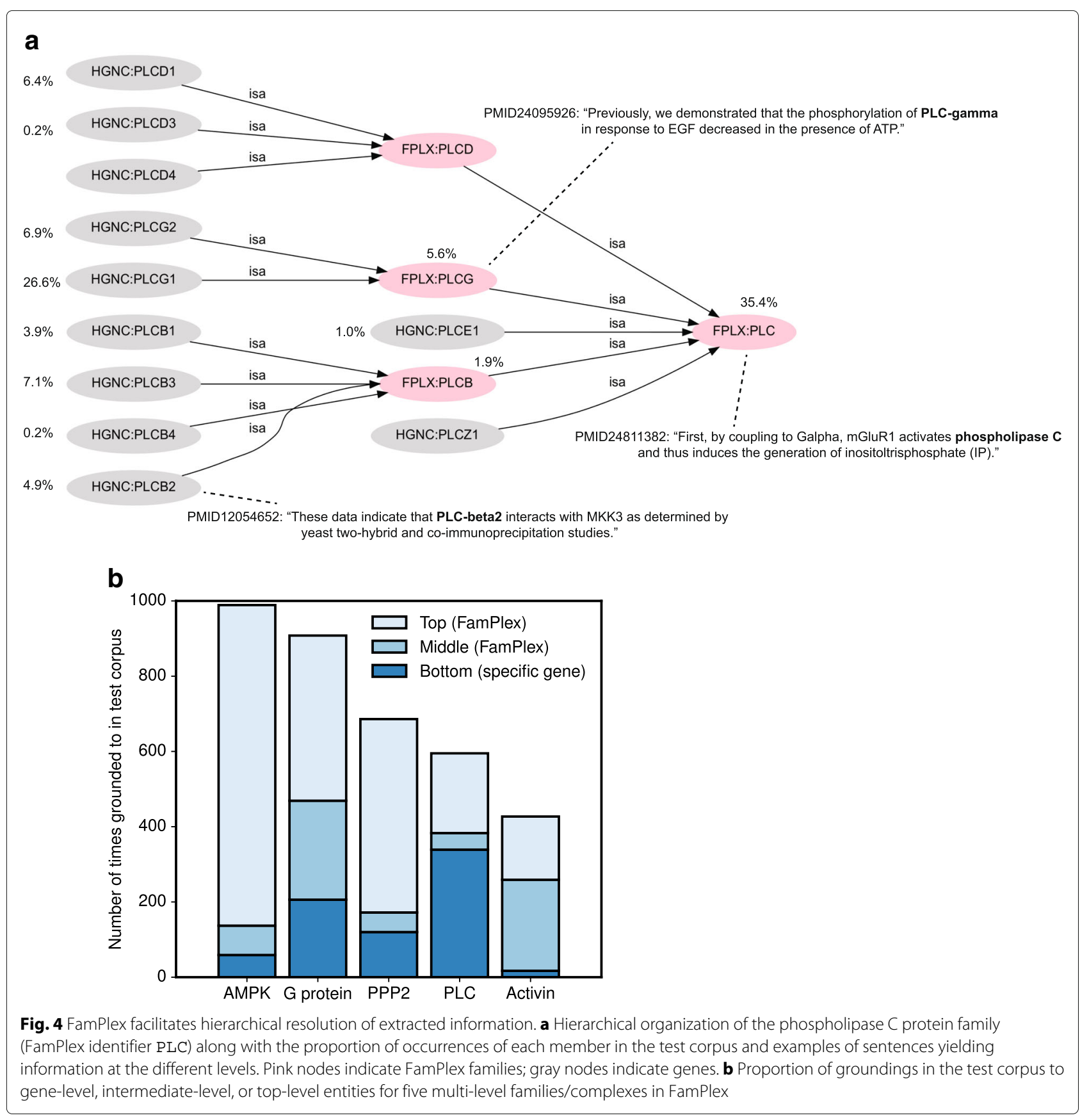

OpenBEL to be the most systematic and reusable, and we therefore drew heavily on OpenBEL in the construction of FamPlex. FamPlex differs from the families and complexes defined in resources such as Reactome, SIGNOR and OpenBEL in three important ways: (i) it includes an extensive set of lexicalizations to assist in grounding, (ii) it enumerates equivalent family/complex identifiers between many of these resources, allowing for mechanistic information to be integrated at the family/complex level, and (iii) it allows for a multi-level entity hierarchy corresponding to the terms and concepts used in the literature.
The third category of related resources are biomedical ontologies such as GO and terminology resources such as NCIT and MeSH. While these resources are the most broadly extensive and often contain synonyms for concepts, they have uneven coverage of protein families and complexes specifically. In addition (as described in our evaluation of grounding to NCIT in the TRIPS reading system) many identifiers representing protein families and complexes do not incorporate child concepts at the gene level, limiting their value for relationship resolution. 
Thus, while FamPlex draws on and provides crossreferences to all three classes of resources described above, it differs from all of them in providing a consistent, multi-level taxonomy of human protein families and complexes that is suitable for grounding and relationship resolution in text mining and biocuration.

\section{Limitations}

The relatively high recall achieved by FamPlex on the Biocreative entity normalization dataset suggests that it provides substantial coverage of relevant protein families, complexes and their lexical synonyms. However, it is not exhaustive. Further empirically-guided curation of the identifiers and grounding map is likely to improve grounding precision and recall still further, and with additional work mappings to other ontologies can be made more comprehensive.

FamPlex does not directly address the problem of ambiguity, selecting among multiple alternative groundings for the same entity. For example, "MEK" can refer to the family of MAPK/ERK Kinases or to the solvent methyl ethyl ketone. Resolving such ambiguities requires an examination of the named entity in the broader context of the sentence or article [30]. However, the use of FamPlex does increase the likelihood that relevant groundings to protein families will not be missed, and can therefore be considered alongside alternative groundings during an ambiguity resolution procedure.

\section{Accessibility and Extensibility}

We chose CSV files as the primary format for FamPlex to maximize accessibility and extensibility. CSV files can be opened and edited in any spreadsheet program or text editor, allowing biologists with no background in literature mining to assist in the curation of the grounding map or create mappings to other resources. Because the files are hosted on GitHub, other users can easily fork and make use-case specific extensions or other contributions that can be merged back into the main repository.

In addition to the CSV files, FamPlex includes an Open Biomedical Ontologies (OBO) [31] export feature to facilitate integration into OBO-based workflows. FamPlex relations and mappings have been integrated into the TRIPS/DRUM reading system [27] via OBO-exported content.

\section{Conclusions}

In this paper we describe the challenge posed by protein families and multi-protein complexes for machine reading of the biomedical literature. We introduce FamPlex, a new lexical and ontological resource that addresses these challenges and improves grounding and relationship resolution in two different reading systems [21, 27]. FamPlex fills a gap in existing bioinformatics resources, linking information about families and complexes in protein and pathway databases to a set of lexical synonyms that occur with high frequency in the scientific literature. Empirical evaluation shows that the hierarchical organization of FamPlex enables the integration of mechanistic information about gene families, complexes, and their individual subunits. This is important because information about biochemical mechanisms is often reported in terms of classes of entities whereas large-scale profiling experiments yield data about individual genes and proteins. FamPlex therefore supports the broader goal of making text mining a key contributor to the process of obtaining biological insight from high throughput omic data by drawing on relevant mechanistic knowledge. We speculate that similar resources for resolving hierarchical relationships among entities could be useful in other domains of machine reading and natural language processing.

\section{Availability and requirements \\ Project name: FamPlex \\ Home Page: https://github.com/sorgerlab/famplex \\ Operating systems: Platform-independent}

Programming language: Not required (helper scripts available in Python 3)

License: Creative Commons CCO

\begin{abstract}
Abbreviations
API: Application programming interface; ATCC: American type culture collection; CSV: Comma-separated variable; DRUM: Deep reader for understanding mechanisms; HGNC: HUGO gene nomenclature committee; HMDB: Human metabolome database; INDRA: Integrated network and dynamical reasoning assembler; MeSH: Medical subject headings; NCBO: National center for biomedical ontology; NCIT: National cancer institute thesaurus; NER: Named entity recognition; OBO: Open biological and biomedical ontology; REACH: Reading and assembling contextual and holistic mechanisms; OpenBEL: Open biological expression language; SIGNOR: Signaling network open resource; TRIPS: The rochester interactive planning system
\end{abstract}

\section{Acknowledgements}

The authors would like to thank Tom Hicks, Mihai Surdeanu and Lucian Galescu for useful discussions and assistance with REACH and TRIPS, and Petar Todorov, Bobby Sheehan, Lily Chylek, Jeremy Muhlich and Isabel Latorre for their contributions to entity curation.

Funding

This work has been supported by DARPA grants W911NF-14-1-0397 and W91 NF-15-1-0544. The funding body did not play a role in the design of the study, the collection, analysis or interpretation of data and in writing the manuscript.

\section{Availability of data and materials}

The datasets generated and analyzed during the current study, as well as the source code used to generate results is available in the repository https:// github.com/sorgerlab/famplex_paper. FamPlex is available at https://github. com/sorgerlab/famplex under the Creative Commons CCO license.

\section{Authors' contributions}

$J A B$ and $B M G$ conceived and implemented the resource. JAB, BMG and PKS wrote the paper. All authors read and approved the final version of the manuscript. 


\section{Ethics approval and consent to participate}

Not applicable.

\section{Competing interests}

The authors declare that they have no competing interests.

\section{Publisher's Note}

Springer Nature remains neutral with regard to jurisdictional claims in published maps and institutional affiliations.

\section{Received: 27 November 2017 Accepted: 17 May 2018}

Published online: 28 June 2018

\section{References}

1. Babur O, Gönen M, Aksoy BA, Schultz N, Ciriello G, Sander C, Demir E. Systematic identification of cancer driving signaling pathways based on mutual exclusivity of genomic alterations. Genome Biol. 2015;16:45. https://doi.org/10.1186/s13059-015-0612-6.

2. García-Campos MA, Espinal-Enríquez J, Hernández-Lemus E. Pathway Analysis: State of the Art. Front Physiol. 2015;6:383. https://doi.org/10. 3389/fphys.2015.00383.

3. Korkut A, Wang W, Demir E, Aksoy BA, Jing X, Molinelli EJ, Babur O, Bemis DL, Sumer SO, Solit DB, et al. Perturbation biology nominates upstream-downstream drug combinations in RAF inhibitor resistant melanoma cells. Elife. 2015:4:04640.

4. Campbell J, Ryan CJ, Brough R, Bajrami I, Pemberton HN, Chong IY, Costa-Cabral S, Frankum J, Gulati A, Holme H, Miller R, Postel-Vinay S, Rafiq R, Wei W, Williamson CT, Quigley DA, Tym J, Al-Lazikani B, Fenton T, Natrajan R, Strauss SJ, Ashworth A, Lord CJ. Large-scale profiling of kinase dependencies in cancer cell lines. Cell Rep. 2016;14(10):2490-501. https://doi.org/10.1016/j.celrep.2016.02.023.

5. Demir E, Cary MP, Paley S, Fukuda K, Lemer C, Vastrik I, Wu G, D'Eustachio P, Schaefer C, Luciano J, Schacherer F, Martinez-Flores I, Hu Z, Jimenez-Jacinto V, Joshi-Tope G, Kandasamy K, Lopez-Fuentes AC, Mi H, Pichler E, Rodchenkov I, Splendiani A, Tkachev S, Zucker J, Gopinath G, Rajasimha H, Ramakrishnan R, Shah I, Syed M, Anwar N, Babur O, Blinov M, Brauner E, Corwin D, Donaldson S, Gibbons F, Goldberg R, Hornbeck P, Luna A, Murray-Rust P, Neumann E, Ruebenacker O, Samwald M, van lersel M, Wimalaratne S, Allen K, Braun B, Whirl-Carrillo M, Cheung K-H, Dahlquist K, Finney A, Gillespie M, Glass E, Gong L, Haw R, Honig M, Hubaut O, Kane D, Krupa S, Kutmon M, Leonard J, Marks D, Merberg D, Petri V, Pico A, Ravenscroft D, Ren L, Shah N, Sunshine M, Tang R, Whaley R, Letovksy S, Buetow KH, Rzhetsky A, Schachter V, Sobral BS, Dogrusoz U, McWeeney S, Aladjem M, Birney E, Collado-Vides J, Goto S, Hucka M, Le Novère N, Maltsev N, Pandey A, Thomas P, Wingender E, Karp PD, Sander C, Bader GD. The BioPAX community standard for pathway data sharing. Nat Biotechnol. 2010;28(9):935-42. https://doi.org/10.1038/nbt.1666.

6. Cerami EG, Gross BE, Demir E, Rodchenkov I, Babur O, Anwar N, Schultz N, Bader GD, Sander C. Pathway Commons, a web resource for biological pathway data. Nucleic Acids Res. 2011;39(Database issue):685-90. https:// doi.org/10.1093/nar/gkq1039.

7. Ananiadou S, Thompson P, Nawaz R, McNaught J, Kell DB. Event-based text mining for biology and functional genomics. Brief Funct Genomics. 2015;14(3):213-30. https://doi.org/10.1093/bfgp/elu015.

8. Morgan AA, Lu Z, Wang X, Cohen AM, Fluck J, Ruch P, Divoli A, Fundel K, Leaman R, Hakenberg J, et al. Overview of BioCreative II gene normalization. Genome Biol. 2008;9(2):3.

9. Pyysalo S, Ohta T, Kim J-D, Tsujii J. Static relations: A piece in the biomedical information extraction puzzle. In: Proceedings of the Workshop on Current Trends in Biomedical Natural Language Processing. BioNLP '09. Stroudsburg: Association for Computational Linguistics; 2009. p. 1-9.

10. Van Landeghem S, Ginter F, Van de Peer $Y$, Salakoski T. EVEX: a PubMed scale resource for homology-based generalization of text mining predictions. In: Proceedings of BioNLP 2011 Workshop. Stroudsburg: Association for Computational Linguistics; 2011. p. 28-37.

11. Björne J, Salakoski T. Tees 2.1: Automated annotation scheme learning in the BioNLP 2013 shared task. In: Proceedings of the BioNLP Shared Task 2013 Workshop. Stroudsburg: Association for Computational Linguistics; 2013. p. 16-25.
12. Blaschke $C$, Hirschman $L$, Valencia A. Information extraction in molecular biology. Brief Bioinform. 2002;3(2):154-65.

13. Tsuruoka Y, McNaught J, Ananiadou S. Normalizing biomedical terms by minimizing ambiguity and variability. BMC Bioinformatics. 2008;9:2.

14. Schuemie MJ, Mons B, Weeber M, Kors JA. Evaluation of techniques for increasing recall in a dictionary approach to gene and protein name identification. J Biomed Inform. 2007;40(3):316-24.

15. Wei C-H, Kao H-Y, Lu Z. GNormPlus: an integrative approach for tagging genes, gene families, and protein domains. BioMed Res Int. 2015;2015.

16. Leaman R, Lu Z. TaggerOne: joint named entity recognition and normalization with semi-Markov models. Bioinformatics. 2016;32(18): 2839-46.

17. Liu H, Christiansen T, Baumgartner WA, Verspoor K. BioLemmatizer: a lemmatization tool for morphological processing of biomedical text. J Biomed Semant. 2012;3(1):3.

18. Van Landeghem S, Björne J, Wei C-H, Hakala K, Pyysalo S, Ananiadou S, Kao H-Y, Lu Z, Salakoski T, Van de Peer Y, et al. Large-scale event extraction from literature with multi-level gene normalization. PloS ONE. 2013;8(4):55814

19. Whetzel PL, Noy NF, Shah NH, Alexander PR, Nyulas C, Tudorache T, Musen MA. BioPortal: enhanced functionality via new Web services from the National Center for Biomedical Ontology to access and use ontologies in software applications. Nucleic Acids Res. 2011;39(Web Server issue):541-5.

20. Valenzuela-Escárcega MA, Hahn-Powell G, Hicks T, Surdeanu M. A domain-independent rule-based framework for event extraction. In: ACL-IJCNLP 2015 - 53rd Annual Meeting of the Association for Computational Linguistics and the 7th International Joint Conference on Natural Language Processing, Proceedings of System Demonstrations. Beijing: Association for Computational Linguistics (ACL); 2015. p. 127-32.

21. Valenzuela-Escarcega MA, Babur O, Hahn-Powell G, Bell D, Hicks T, Noriega-Atala E, Wang X, Surdeanu M, Demir E, Morrison CT. Large-scale automated reading with Reach discovers new cancer driving mechanisms. In: Proceedings of the Sixth BioCreative Challenge Evaluation Workshop. Bethesda; 2017. p. 201-3.

22. Fabregat A, Sidiropoulos K, Garapati P, Gillespie M, Hausmann K, Haw R, Jassal B, Jupe S, Korninger F, McKay S, Matthews L, May B, Milacic M, Rothfels K, Shamovsky V, Webber M, Weiser J, Williams M, Wu G, Stein L, Hermjakob H, D'Eustachio P. The Reactome pathway Knowledgebase. Nucleic Acids Res. 2016;44(D1):481-7. https://doi.org/10.1093/nar/ gkv1351.

23. The OpenBEL Consortium. OpenBEL Framework Resources. 2017. https:// github.com/OpenBEL/openbel-framework-resources. Accessed 26 May 2018.

24. Maglott D, Ostell J, Pruitt KD, Tatusova T. Entrez Gene: gene-centered information at NCBI. Nucleic Acids Res. 2005;33(Database Issue):54-8. https://doi.org/10.1093/nar/gki031.

25. Manning CD, Surdeanu M, Bauer J, Finkel JR, Bethard S, McClosky D. The Stanford CoreNLP natural language processing toolkit. In: ACL (System Demonstrations). Stroudsburg: Association for Computational Linguistics; 2014. p. 55-60.

26. Gyori BM, Bachman JA, Subramanian K, Muhlich JL, Galescu L, Sorger PK From word models to executable models of signaling networks using automated assembly. Mol Syst Biol. 2017;13(11):954. https://doi.org/10. 1101/119834.

27. Allen J, de Beaumont W, Galescu L, Teng CM. Complex Event Extraction using DRUM. ACL-IJCNLP 2015. 2015;1:1-11.

28. Navarro G. A guided tour to approximate string matching. ACM Comput Surv. 2001;33(1):31-88. https://doi.org/10.1145/375360.375365.

29. Liechti R, George N, Götz L, El-Gebali S, Chasapi A, Crespo I, Xenarios I, Lemberger T. SourceData: a semantic platform for curating and searching figures. Nat Methods. 2017;14(11):1021.

30. Okazaki N, Ananiadou S. Building an abbreviation dictionary using a term recognition approach. Bioinformatics. 2006;22(24):3089-95.

31. Smith B, Ashburner M, Rosse C, Bard J, Bug W, Ceusters W, Goldberg LJ, Eilbeck K, Ireland A, Mungall CJ, Leontis N, Rocca-Serra P, Ruttenberg A, Sansone S-A, Scheuermann RH, Shah N, Whetzel PL, Lewis S. The OBO Foundry: coordinated evolution of ontologies to support biomedical data integration. Nat Biotechnol. 2007;25(11):1251. https://doi.org/10.1038/ nbt1346. 